\title{
Inhibition of the methyltranferase EZH2 improves aortic performance in experimental thoracic aortic aneurysm
}

\author{
Christian L. Lino Cardenas, ${ }^{1,2,3}$ Chase W. Kessinger, ${ }^{2,3}$ Carolyn MacDonald, ${ }^{1,2,3}$ Arminder S. Jassar, ${ }^{1,4}$ \\ Eric M. Isselbacher, ${ }^{1,2,3}$ Farouc A. Jaffer, ${ }^{2,3}$ and Mark E. Lindsay, ${ }^{1,2,3}$ \\ ${ }^{1}$ Thoracic Aortic Center, ${ }^{2}$ Cardiovascular Research Center, ${ }^{3}$ Cardiology, Department of Medicine, ${ }^{4}$ Division of Cardiothoracic \\ Surgery, Department of Surgery, and ${ }^{5}$ Pediatric Cardiology, Department of Pediatrics, Massachusetts Ceneral Hospital \\ (MCH), Harvard Medical School, Boston, Massachusetts, USA.
}

\begin{abstract}
Loss-of-function mutations in genes encoding contractile proteins have been observed in thoracic aortic aneurysms (TAA). To gain insight into the contribution of contractile protein deficiency in the pathogenesis of TAA, we examined human aneurysm samples. We found multiple contractile gene products deficient in TAA samples, and in particular, expression of SM22 $\alpha$ was inversely correlated with aneurysm size. SM22 $\alpha$-deficient mice demonstrated pregnancy-induced aortic dissection, and SM22 $\alpha$ deficiency worsened aortic aneurysm in Fbn $1^{\mathrm{C1039C/+}}$ (Marfan) mice, validating this gene product as a TAA effector. We found that repression of SM22 $\alpha$ was enforced by increased activity of the methyltransferase EZH2. TCF- $\beta$ effectors such as SMAD3 were excluded from binding SM22 $\alpha$-encoding chromatin (TAGLN) in TAA samples, while treatment with the EZH2 inhibitor CSK343 improved cytoskeletal architecture and restored SM22 $\alpha$ expression. Finally, inhibition of EZH2 improved aortic performance in Fbn $7^{\mathrm{C} 1039 \mathrm{C} /+}$ mice, in association with restoration of contractile protein expression (including SM22 $\alpha$ ). Together, these data inform our understanding of contractile protein deficiency in TAA and support the pursuit of chromatin modifying factors as therapeutic targets in aortic disease.
\end{abstract}

Conflict of interest: The authors have declared that no conflict of interest exists.

Submitted: September 14, 2017 Accepted: January 25, 2018 Published: March 8, 2018

Reference information: JCI Insight. 2018;3(5):e97493. https:// doi.org/10.1172/jii.insight.97493

\section{Introduction}

Contractile proteins are vital components of the vascular smooth muscle cell (VSMC) cytoskeleton and perform kinetic and static regulatory functions in both health and disease. Loss of contractile protein expression has been observed in various forms of vascular disease including aneurysms (1-3), atherosclerosis (4-6), and vascular stenosis $(7,8)$. Such observations may be more than correlative, as multiple genes involved in the VSMC contractile apparatus have been implicated in familial forms of thoracic aortic aneurysm (TAA). For instance, pathogenic variation in the genes ACTA2 and MYH11 encoding the complementary components of the smooth muscle actin-myosin (SMA-myosin) apparatus, $\alpha$-SMA and smooth muscle myosin heavy chain (smMHC), both have been shown to cause TAA in humans $(9,10)$. Additionally, regulators of this interaction have been implicated in TAA, including loss-of-function mutations in $M Y L K$ encoding myosin light chain kinase and a recurrent gainof-function mutation in PRKG1, a negative regulator of myosin light chain phosphatase $(11,12)$. These genetic data directly implicate gene products involved in VSMC contraction in the pathogenesis of TAA. Disruption in a second group of matrix-independent gene products have been implicated in TAA. Syndromic presentations (e.g., Loeys-Dietz syndrome, Aneurysms-Osteoarthritis syndrome, or Marfan syndrome) have been described with loss-of-function mutations in genes encoding various members of the TGF- $\beta$ signaling cascade, including TGFBR1, TGFBR2, TGFB2, TGFB3, and SMAD3, among others (13-17). These genes encode TGF- $\beta$ ligands (TGFB2/3), TGF- $\beta$ receptors (TGFR1/2), and downstream mediators of canonical TGF- $\beta$ signaling (such as SMAD3). Both syndromic and nonsyndromic presentations of TAA affect similar anatomic portions of the aorta and show similar disruption of tissue architecture, suggesting a shared pathogenesis (18).

The expression of contractile protein elements such as $\alpha$-SMA, SM22 $\alpha$, and smoothelin can be induced in experimental contexts by exogenously applied TGF- $\beta$ (19-22), leading to models whereby loss-of-function genetic variation in the canonical TGF- $\beta$ signaling cascade may lead directly to deficiency of contractile elements. However, increased levels of TGF- $\beta$ signaling, as assessed by phosphorylation of Smad pro- 
teins ( $\mathrm{p}-\mathrm{SMAD} 2 / 3$ ) have routinely been observed in both genetically triggered and sporadic aortic samples $(18,23)$. Despite increased TGF- $\beta$ signaling, contractile protein elements in aortic specimens from TAA are often deficient, and antagonism of TGF- $\beta$ signaling has been shown to be therapeutic in noninflammatory experimental TAA (24). These observations suggest a discrepancy between transcriptional circuits operating in VSMCs in health versus those in TAA.

In this study, we analyzed human aneurysm samples to examine proteins of the contractile apparatus in TAA. Multiple gene products are deficient in human and murine TAA samples and, in particular, expression of the protein SM22 $\alpha$. We first demonstrated that loss of SM22 $\alpha$ is a direct effector of aneurysm progression and aortic dissection in vivo. Investigation of SM22 $\alpha$ transcriptional regulation revealed hypermethylation of consensus sequences containing SMAD-transcription factor binding sites (TFBS) at the locus of the TAGLN (SM22 $\alpha$-encoding) gene in human aneurysm tissue associating with histone 3 lysine 27 trimethylation (histone 3 lysine 27 trimethylation [H3K27me3]) modifications. Targeting the methyltransferase EZH2 (catalyzing H3K27me3 modifications) with the small molecule inhibitor GSK343 improved aortic performance in

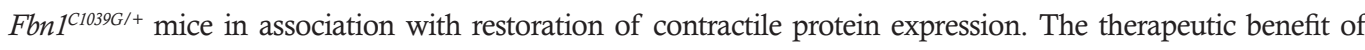
GSK343 inhibitor was comparable to losartan; however, these compounds exhibited distinct molecular mechanisms. These data illuminate the critical role of VSMC contractile proteins in aortic homeostasis, describe potentially novel epigenetic modification in thoracic aortic disease, and expand the therapeutic options for TAA.

\section{Results}

Contractile protein expression is associated with TAA pathology. Contractile protein downregulation has been observed in various forms of vascular disease. To study how this phenomenon may relate to disease progression in TAA, we choose to evaluate transcript expression of previously described markers of the VSMC phenotypic state (25) in aortic tissue taken at the time of surgery. Several genes indicative of the VSMC synthetic state were upregulated in comparison with control aortas. Conversely, transcripts encoding the contractile proteins were significantly deficient in tissue from TAA repair and patients who have experienced aortic dissection (Figure 1A and Supplemental Table 1; supplemental material available online with this article; https://doi.org/10.1172/jci.insight.97493DS1). The TAGLN gene, encoding SM22 $\alpha$ in particular, has been reported to be downregulated (Fold Change $[F C\}=0.12)$ in patients with aortic dissection $(26,27)$, and we therefore chose to examine this factor further. Expression of SM22 $\alpha$ protein in aneurysm samples collected from patients with TAA at the time of cardiac surgery demonstrate significant repression from patients with syndromic and/or nonsyndromic aortic disease (Figure 1B). Examination of human TAA samples via IHC similarly demonstrates that the decrement in contractile proteins localize to the aortic media (Figure 1C). To study SM22 $\alpha$ regulation in a more controlled system, we examined expression in the Fbn $1^{\text {C1039G/+ }}$ mouse model of Marfan syndrome and found a similar repression at both mRNA and protein level (Figure 1, D and E). Interestingly, the expression of SM22 $\alpha$ was found to be closely and inversely correlated with aneurysm size (Figure 1F). These observations led us to examine the effect of SM22 $\alpha$ deficiency on VSMC behavior. Targeting SM22 $\alpha$ expression with siRNA-mediated silencing induced cytoskeletal destabilization and vacuole formation (Figure 1, G and $\mathrm{H}$ ), 2 cellular phenotypes directly visualized in classic electron microscopic examination of TAA tissue from human patients (28). Furthermore, matrix metalloproteinase (MMP) activity known to be associated with aortic aneurysms was activated in SM22 $\alpha$ siRNA-treated VSMCs (Figure 1I), consistent with previous reports of SM22 $\alpha$ as a negative regulator of MMP expression (29).

$S M 22 \alpha$ is required for aortic homeostasis. These data suggest that loss of SM22 $\alpha$ expression may contribute to the aneurysm progression in TAA. To examine this concept, we crossed SM22 $\alpha$-deficient mice (Tagln ${ }^{-/-}$or $\mathrm{Sm} 22^{--}$) to $\mathrm{Fbn} 1^{\mathrm{Cl039G/+}}$ mice to generate SM22 $\alpha$-deficient $\mathrm{Fbn} 1^{\mathrm{Cl039G/+}}$ animals and examined aortic aneurysm progression. Consistent with the role of SM22 $\alpha$ as an important determinant of aortic homeostasis, Fbn $1^{C 1039 G /+}$ $\operatorname{Sm} 22^{-/-}$mice exhibited larger aortas and more rapid aortic growth than age-matched $\mathrm{Fbn} \mathrm{C}^{\mathrm{C} 1039 \mathrm{G} /+} \mathrm{Sm} 22^{+/+}$mice (Figure 2, A and B). Histochemical examination of $S m 22^{-1-}$ aortas revealed increased levels of collagen deposition in the adventitia when compared with WT mice (Figure 2C). In Fbn $1^{\mathrm{ClO3gG/+}} \mathrm{Sm} 22^{-/-}$mice, collagen deposition was further accentuated and aortic wall architecture more disrupted than age-matched $\mathrm{Fbn} \mathrm{C}^{\mathrm{C} 1039 \mathrm{G} /+} \mathrm{Sm} 22^{+/+}$ mice (Figure 2C). These changes were accompanied by increased MMP activity in Fbn $1^{\mathrm{ClO} 39 \mathrm{G} /+} \mathrm{Sm} 22^{-/-}$mice when compared with $\mathrm{Fbn} 1^{\mathrm{ClO} 39 \mathrm{G} /+} \mathrm{Sm} 22^{+/+}$mice (Figure 2D).

Examination of aortas from $S m 22^{-1-}$ demonstrated only mild, nonsignificant aortic enlargement when compared with $S m 22^{+/+}$mice (Figure 2B). However, during the breeding, we noted premature lethality in $\operatorname{Sm} 22^{-/}$dams. Postmortem examination of pregnant mice demonstrated evidence of hemothorax caused 
A

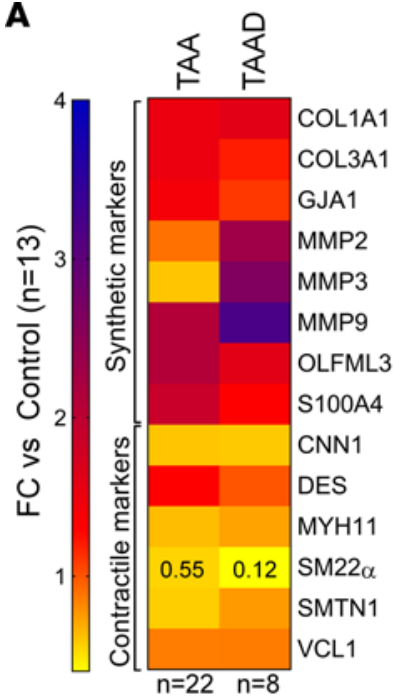

B

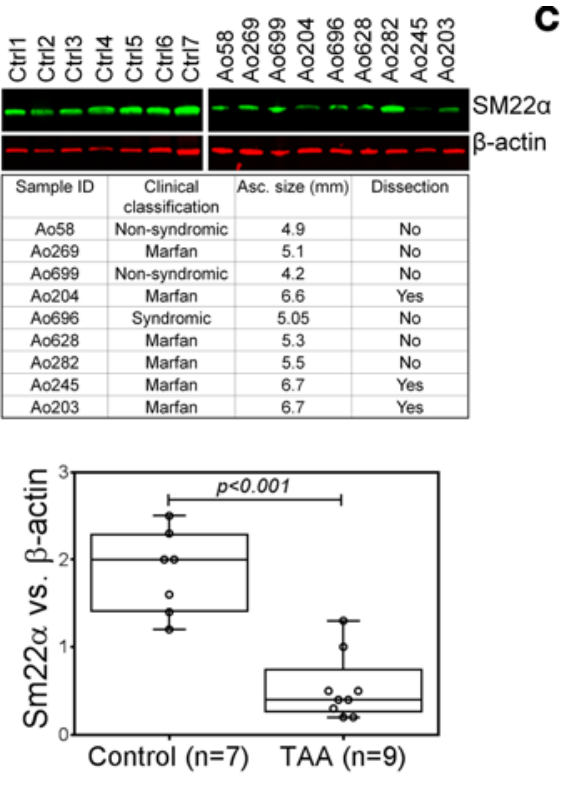

C

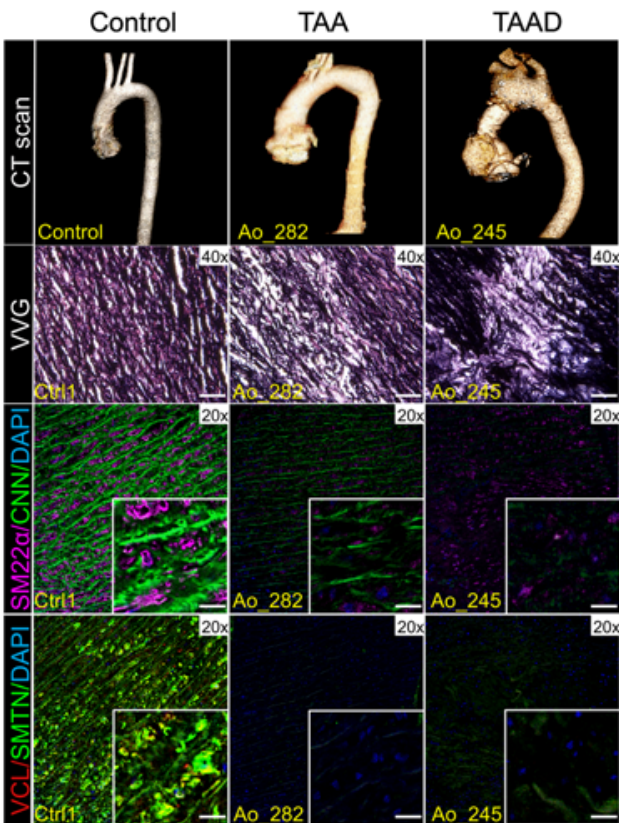

G

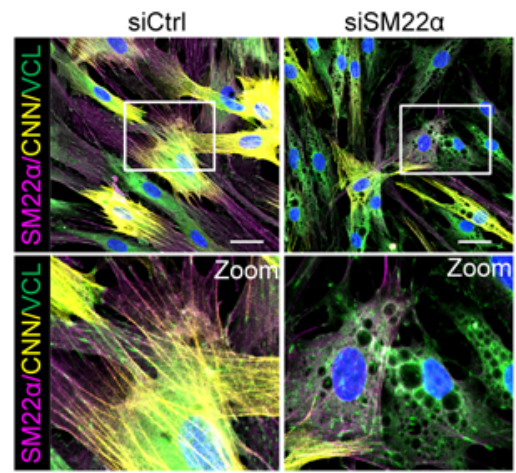

$\mathbf{H}$
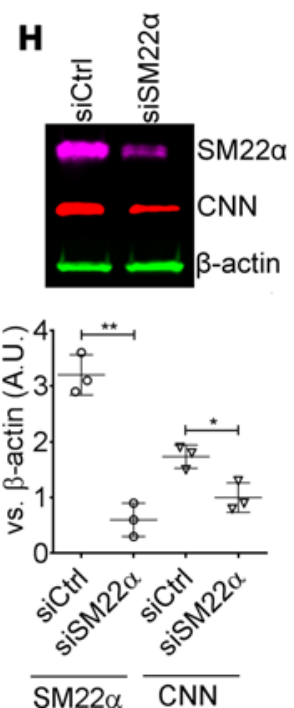
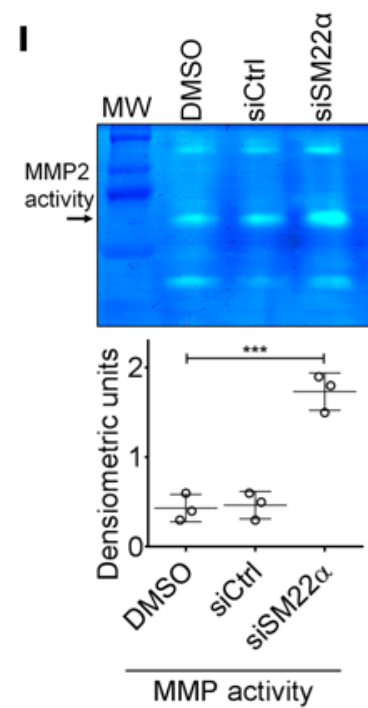

Figure 1. Decrement of members of the VSMC contractile apparatus in patients with TAA. (A) Expression pattern of VSMC phenotypic markers in human aortic tissue from control $(n=13)$ and TAAD $(n=30)$ donors. Loss of TAGLN (SM22 $\alpha)$ transcript were more significant in patients who have experienced dissection (TAA FC $=0.55$ vs TAAD FC $=0.12$ ). (B) Immunoblotting analysis of SM22 $\alpha$ protein in human aortic tissue from control $(n=7)$ and TAAD $(n=9)$ donors. (C) Anatomical reconstruction of patient images and immunofluorescence staining of contractile proteins of human aortas from control, TAA, and TAAD samples (top row). Verhoeff-Van Gieson stain (VVG) staining of aortic samples from patients demonstrates elastin fragmentation. Scale bar: $150 \mu \mathrm{m}$ (second row). Immunofluorescence from aortic samples showing SM22 $\alpha$ (magenta), calponin (Cnn) (green), DAPI (blue), vinculin (red), smoothelin (green), and DAPI (blue). Scale bar: $50 \mu \mathrm{m}$, inset zoom 3-fold magnification. (D) Representative photomicrographs of latex-injected WT and Fbn ${ }^{110396 /+}$ mouse 
hearts and ascending aortas. Red arrows indicate the ascending portion of the aorta. Aortic elastin fibers were stained with Verhoeff-Van Gieson stain (VVG), and immunofluorescence of DAPI (blue), F-actin (green), and Sm22 $\alpha$ (magenta). Scale bar: $50 \mu m$. (E) qPCR analysis of Tagln (Sm22 $\alpha$ ) transcript in aortic tissue and plasma from WT $(n=7)$ and Fbn $7^{110396 /+}(n=7) 6$-month-old mice. (F) Correlation of Tagln (Sm22 $\left.\alpha\right)$ tissue expression with aortic dimensions in Fbn1 $1^{C 10396 /+}$ aortas $(n=7)$ (least squares method used for curve fit). (G) Immunofluorescence staining of contractile proteins in aortic VMSC treated with siCtrl or siSM22 $\alpha$. Scale bar: $10 \mu \mathrm{m}$, zoom 3-fold magnification. (H) Immunoblotting analysis of SM22 $\alpha$ inhibition and loss of its binding partner Cnn in aortic VMSC. Quantitation versus $\beta$-actin shown below. (I) siSM $22 \alpha$ treatment of healthy VSMCs induce MMP activity as assessed by in vitro gelatin zymography. ( ${ }^{*} P<0.05$, ${ }^{* *} P<0.01$, ${ }^{* *} P<0.001$, student's $t$ test, 2 tailed). FC, fold change; TAA, Thoracic aortic aneurysm; TAAD, Thoracic aortic aneurysm and dissection; Ctrl, control; SMTN, smoothelin; VCL, vinculin.

by aortic rupture, suggesting a susceptibility of the $S m 22^{-1-}$ aorta to pregnancy-induced aortic disease (Figure $2 \mathrm{E}$ ). Lethality predominantly occurred during the terminal stages of pregnancy, similar to pregnancy-induced aortic dissection in humans (Figure 2F). Examination of postmortem aortic tissue demonstrated increased collagen deposition across the aortic media (Figure 2G).

Inhibition of EZH2 activity improves SM22 $\alpha$ expression in cellular models. These data support the concept that SM22 $\alpha$ deficiency represents a critical mediator of TAA pathogenesis. To examine the transcriptional regulation of the SM22 $\alpha$ (TAGLN gene), we chose to examine the chromatin state directly from human aortic aneurysm samples. By using ChIP quantitative PCR (qPCR) assays, we demonstrate that loss of SM22 $\alpha$ is caused via an increased activity of the methyltransferase EZH2, the catalytic subunit of the PRC2 complex, at the promoter and gene body of the TAGLN gene. We also found a robust enrichment of $\mathrm{H} 3 \mathrm{~K} 27 \mathrm{me} 3$, the catalytic product of $\mathrm{EZH} 2$, in 2 regions within intron 1 (In1.3 and In1.6) (Figure 3A). We therefore analyzed the primary sequence of these 2 regions for possible TFBS using Genomatix (Figure 3B). We identified putative binding sites for SMAD3, Myocardin, KLF factors, and MEF2 via bioinformatics profiling. Due to the well-known association of TAA with TGF- $\beta$ signaling, we chose to examine SMAD3 activity in relation to SM22 $\alpha$ expression. Silencing of SMAD3 in VSMCs revealed that both basal and TGF- $\beta$-induced SM22 $\alpha$ expression are mediated in part by SMAD3 expression (Figure 3C). We therefore examined SMAD3 binding to the In1.3 and In1.6 intronic regions in the presence of exogenously added TGF- $\beta$. Interestingly, SMAD3 binding was detected in control VSMC cultures but not in VSMCs cultured from TAA samples (Figure 3D). The inability of SMAD3 to access these promoter regions correlates with an inability of TGF- $\beta$ to induce TAGLN transcript expression (Figure $3 \mathrm{E})$. Immunofluorescent staining in TAA aortas showed increased levels of H3K27me3 marks (Figure 3F). We therefore tested the ability of GSK343, a potent inhibitor of EZH2, to influence SMAD3-mediated SM22 $\alpha$ transcriptional regulation. Indeed, isolated TAA VSMCs treated with GSK343 inhibitor recovered the ability to induce SM22 $\alpha$ expression after stimulation with recombinant TGF- $\beta 1$ (Figure 3G).

Immunostaining in the murine aorta showed increased levels of $\mathrm{H} 3 \mathrm{~K} 27 \mathrm{me} 3$ and increased colocalization of Ezh2 and H3K27me3 in Fbn $1^{\text {Clo3gG/+ }}$ versus WT mice, (Figure 3H and Supplemental Figure 1A). To determine if Ezh2 may be silencing genes related to aortic homeostasis, such as SM22 $\alpha$, we studied VSMCs isolated from a murine line capable of Ezh2 deletion crossed to Fbn $1^{\mathrm{Cl039G/+}}$ mice. Strikingly, the inability of TGF- $\beta$ to induce SM22 $\alpha$ expression in $\mathrm{Fbn}^{\mathrm{C1039G/+}}$ cells was recovered in cells from $\mathrm{Fbn}^{\mathrm{C1039G/+}}$ mice in which Ezh2 had been deleted (Figure 3, I and J, and Supplemental Figure 1B). Consistent with this finding, overexpression of Ezh2 in VSMCs suppressed SM22 $\alpha$ expression (Supplemental Figure 1, C and D). In addition to recovery of SM22 $\alpha$ expression, inhibition of Ezh2 enhanced TGF- $\beta$-induced stress fiber formation (Figure 3K and Supplemental Figure 1E).

Inhibition of $P R C 2$ activity ameliorates experimental TAA progression. Increased TGF- $\beta$ activity as assayed by phosphorylation of smad proteins and increased ligand expression has been reported repeatedly in TAA tissue (18). We reasoned that contractile genes important for aortic homeostasis (such as TAGLN) that may be induced by TGF- $\beta$ activity in health are repressed through epigenetic modification in $\mathrm{Fbn}^{\mathrm{Cl039G/+}}$ murine aorta. The ability of GSK343 to restore TGF- $\beta$-mediated SM22 $\alpha$ expression in cell culture prompted us to examine EZH2 inhibition as a therapeutic strategy in experimental aneurysms. To address this concept, we treated $\mathrm{Fbn}^{\mathrm{ClO} 103 \mathrm{G} /+}$ mice with either the angiotensin-2 type 1 receptor blocker, losartan, or GSK343 from 2-6 months of life. Activity of GSK343 compound was verified through examination of tissue levels of H3K27me3 (Supplemental Figure 2). Both losartan and GSK343 demonstrated the ability to improve aortic dimensions in $\mathrm{Fbn}^{\mathrm{C} 1039 \mathrm{G} / \mathrm{H}}$ mice (Figure 4A). Examination of VSMC phenotypic markers demonstrated a distinct molecular mechanism for each agent. Losartan treatment showed a better ability to decrease the well-known pathologic markers, including Col1a1, Col3a1, Gja1, and Mmp2 (synthetic markers) (Figure 4B), and improve elastin integrity overall (Figure 4C). While the GSK343 inhibitor showed a better recovery of contractile elements but not a significant improvement in elastin fiber integrity — possibly due to 
A
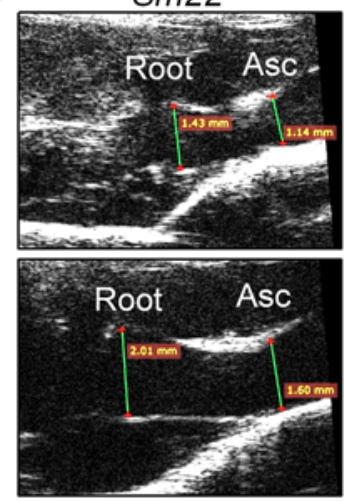

Fbn $1 \mathrm{C} 1039 \mathrm{G} /+^{\mathrm{S}} \mathrm{Sm} 22^{+/+}$
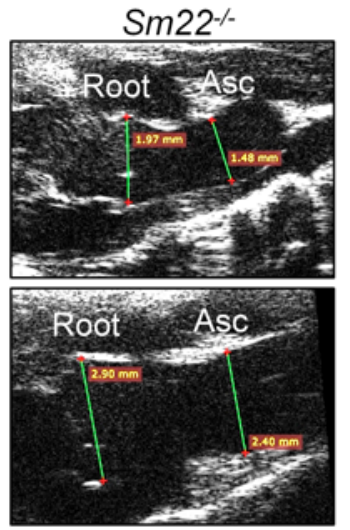

Fbn1 1 1039G/+:Sm22 $\%$

B

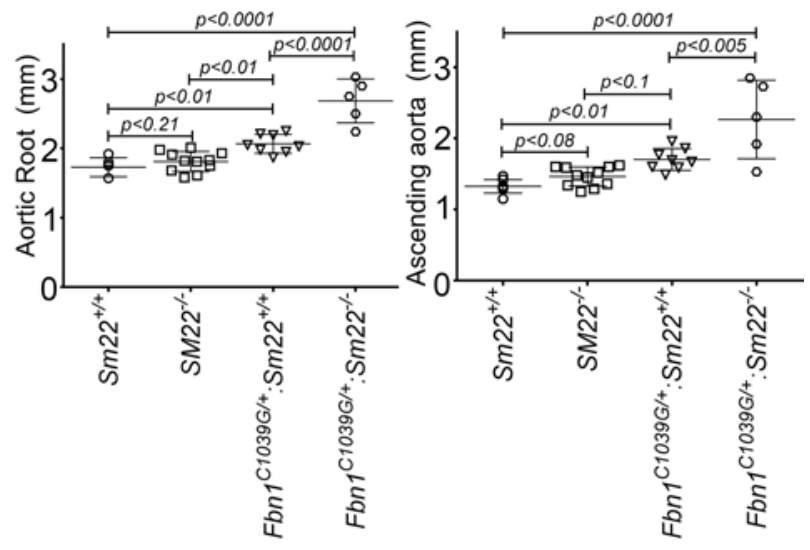

C
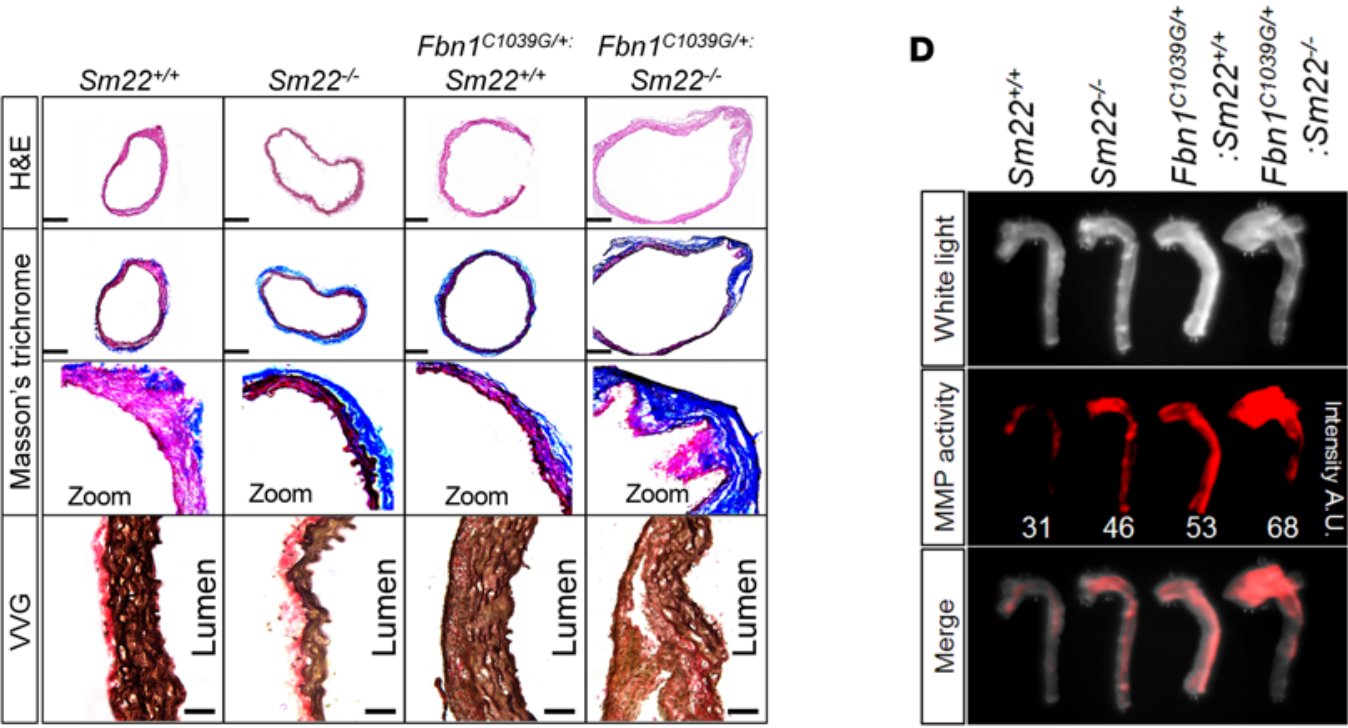

E

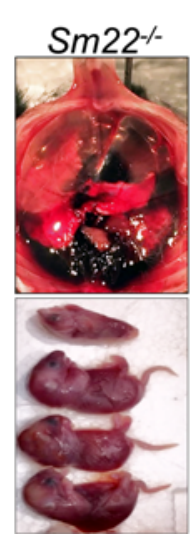

$\mathbf{F}$

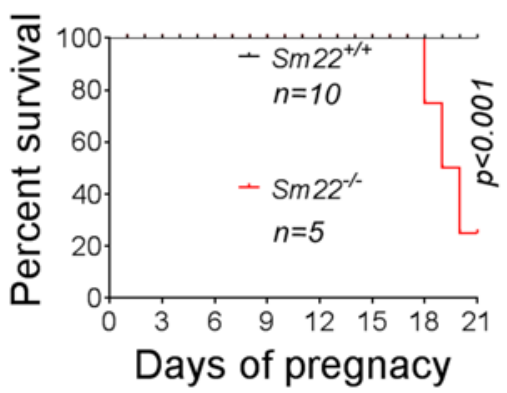

G
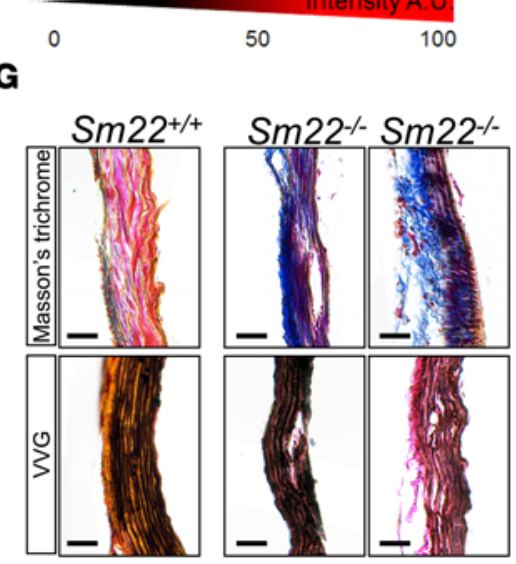

Post-mortem

Figure 2. Loss of SM22 $\alpha$ is a direct effector of aneurysm progression and dissection. (A) Echocardiographic aortic root and ascending aortic quantification of WT and Fbn $7^{10396 /+}$ mice at 4 months of age. (B) Quantification of aortic dimension of $\operatorname{Sm} 22^{+/+}(n=8), S m 22^{-/-}(n=10), F b n 1^{C 10396 /+} \operatorname{Sm} 22^{+/+}(n=10)$, and Fbn $7^{10396 /+} \operatorname{Sm} 22^{-/-}(n=5)$ at 4 months of age (1-way ANOVA, post hoc Tukey's test). (C) Histologic analysis shows abnormal aortic architecture and increased collagen deposition in 4-month-old Sm22-deficient mice; H\&E scale bar: $250 \mu \mathrm{m}$; Masson's trichrome scale bar: $250 \mu \mathrm{m}$ (zoom 5x); VVG scale bar: $50 \mu \mathrm{m}$. (D) Increased MMP activity in 4-month-old Sm22 $\alpha$-deficient mice. (E) Postmortem examination of pregnant mouse shows hemothorax. (F) Kaplan-Meier plot of Sm22 $\alpha$ survival during pregnancy $\left(S \mathrm{~m} 22^{+/+}, n=10\right.$, and $S \mathrm{~m} 22^{-/-}, n=5$, log-rank test) (G) Trichrome and VVG staining of aortas from pregnant $S \mathrm{~m} 22^{+/+}$and $S m 22^{-/-}$mice. Scale bar: $50 \mu \mathrm{m}$., VVG, Verhoeff-Van Gieson stain. 
A

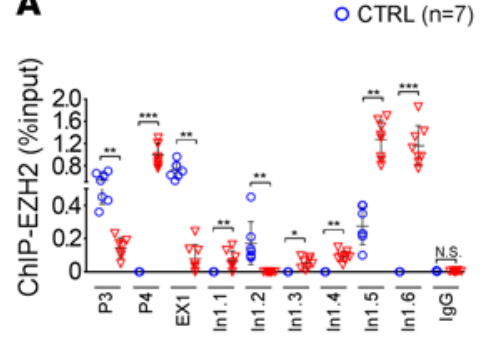

H
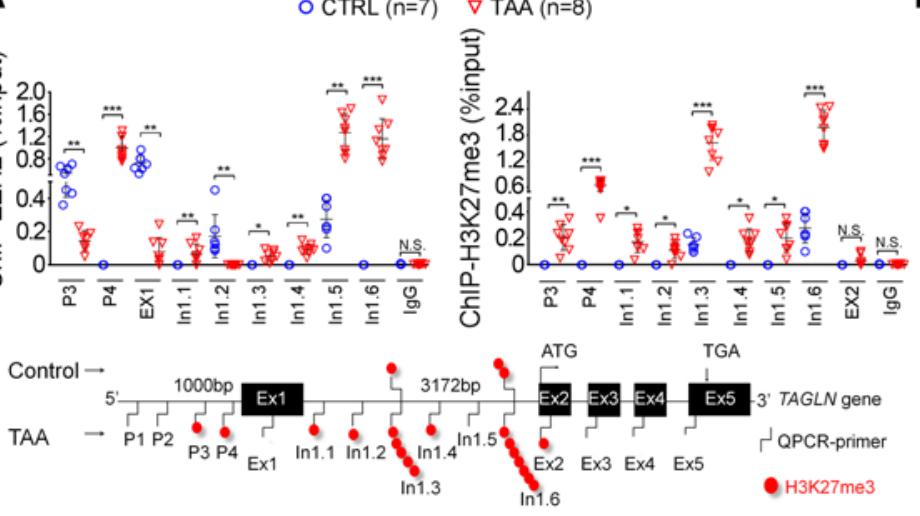

B

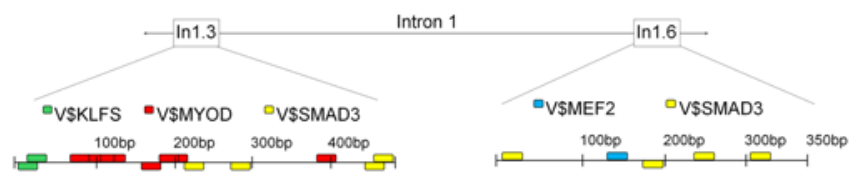

C

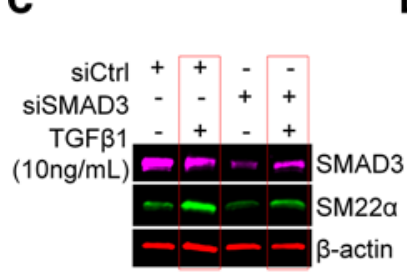

$\mathbf{F}$

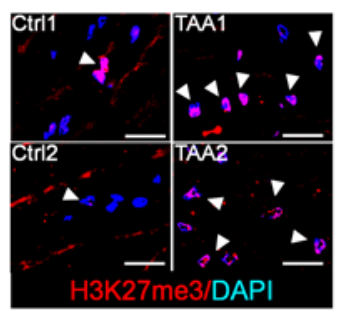

$63 \mathrm{X}$

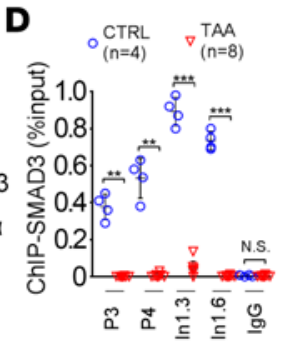

G TGFB1 (10ng/mL)

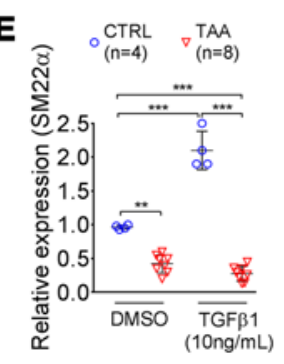

Control 1 TAA 1 Control 2 TAA 2
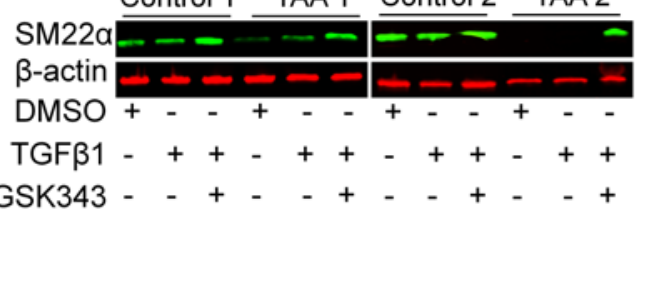
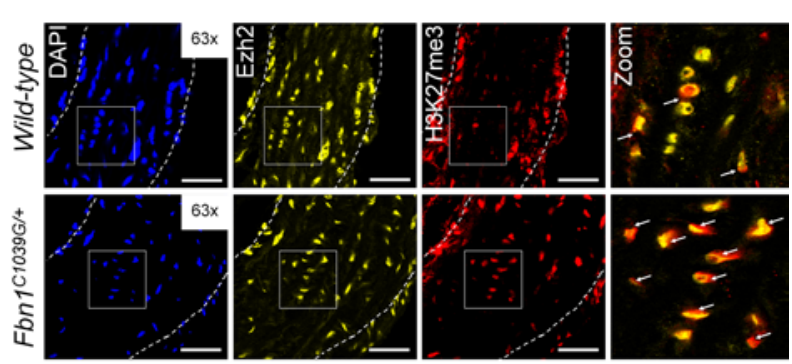

I
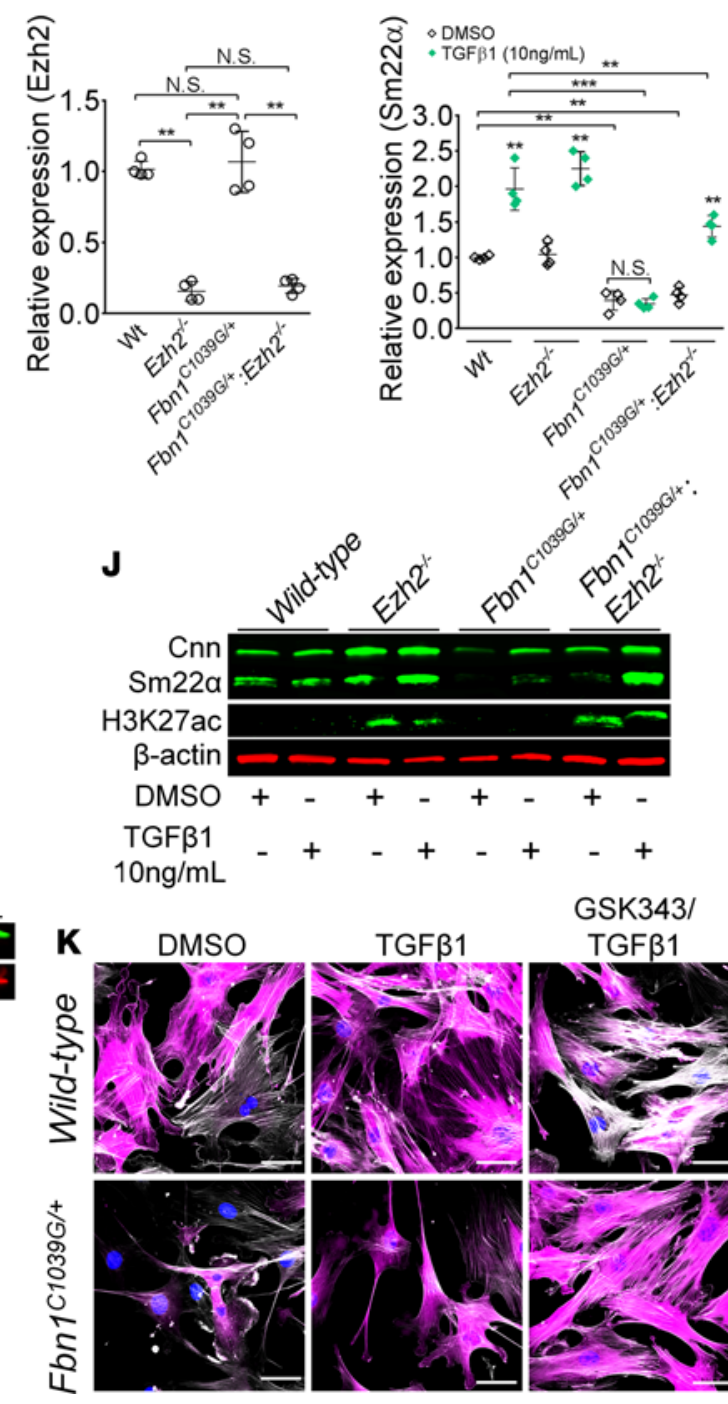

GSK343/

TGF $\beta 1$

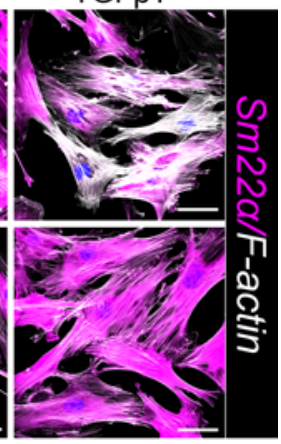

Figure 3. Inhibition of the methyltransferase, EZH2 restores SM22 $\alpha$ expression. (A) ChIP-qPCR assays in human aortic tissue shows increased interaction of EZH2 protein and H3K27me3 modifications in TAA tissue $(n=8)$ when compared with control aortas $(n=7)$ at the TAGLN gene locus. Lower panel shows map of H3K27me3 modifications. ( ${ }^{*} P<0.05$, ${ }^{* *} P<0.01$, ${ }^{* * *} P<0.001$, student's $t$ test versus WT, 2 tailed) (B) Identification of transcription factor binding sides in hypermethylated region within intron 1 using Genomatix. V\$, vertebrates matrix family. (C) VSMCs treated with siSMAD3 demonstrate inhibition of basal and TGF- $\beta$-induced SM22 $\alpha$ expression. (D) SMAD3 ChIP-qPCR assays in VSMCs cultured from TAA $(n=8)$ and treated with TCF- $\beta 1$ (10 $\mathrm{ng} / \mathrm{ml}$ ) demonstrate decreased SMAD3 binding at TAGLN locus when compared with control VSMC cultures $(n=4)\left({ }^{* *} P<0.01,{ }^{* * *} P<0.001\right.$, student's $t$ test versus WT, 2 tailed). (E) qPCR analysis of SM22 $\alpha$ transcript in control $(n=4)$ or TAA $(n=8)$ isolated VSMC cultures treated with recombinant TCF- $\beta 1$ $(10 \mathrm{ng} / \mathrm{ml})\left(^{* *} P<0.01,{ }^{* *} P<0.001,1\right.$-way ANOVA). (F) Immunofluorescent staining of H3K27me3 modifications in human tissue from control and TAA aortas. Scale bar: $20 \mu \mathrm{m}$. Arrows indicate positive H3K27me3 nuclei. (C) Immunoblotting analysis of SM22 $\alpha$ in isolated VSMCs from control and TAA VSMC cultures treated with EZH2 inhibitor (GSK343 $10 \mu \mathrm{M})$ and/or TCF- $\beta 1(10 \mathrm{ng} / \mathrm{ml})$. (H) Immunofluorescent staining of Ezh2 (yellow) and H3K27me3 (red) in 6-month-old Fbn $7^{C 1039 C /+}$ and WT mouse aortas. Scale bar: $40 \mu \mathrm{m}$. (I) qPCR analysis of Ezh2 and Tagln (Sm22 $\alpha$ ) transcripts in mouse VSMCs isolated from WT, Ezh2 $2^{-/}$, Fbn $1^{110396 /+}$, and Fbn $1^{C 10396 /+}$ Ezh2 $2^{-/-}$cells. ${ }^{* *} P<0.01,{ }^{* * *} P<0.001,1$-way ANOVA) (J) Immunoblotting analysis of Sm22 $\alpha$ in mouse VSMCs

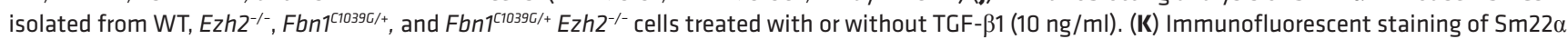
(magenta) and F-actin (gray) in Fbn 1 $1039 \mathrm{C} /+$ and WT mouse VSMCs treated with EZH2 inhibitor (CSK343 $10 \mu \mathrm{M})$ and TGF- $\beta 1$ (10 ng/ml). Scale bar: $10 \mu \mathrm{m}$. TAA, thoracic aortic aneurysm; Ctrl, control; H3K27me3, Histone 3 lysine 27 trimethylation; H3K27ac, Histone 3 acetylated lysine 27. 
A
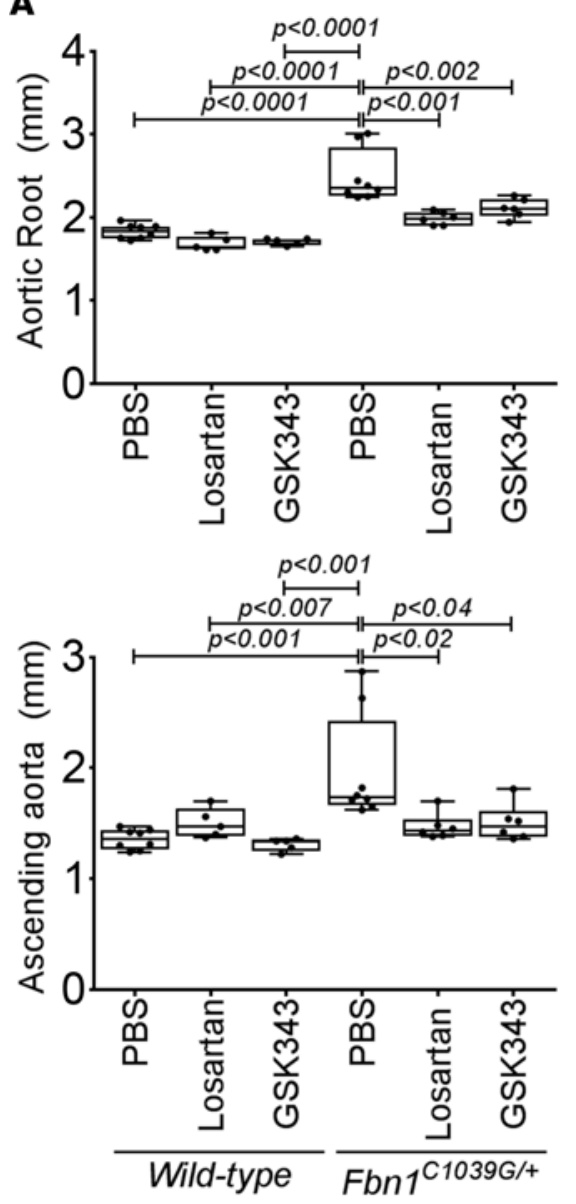

B

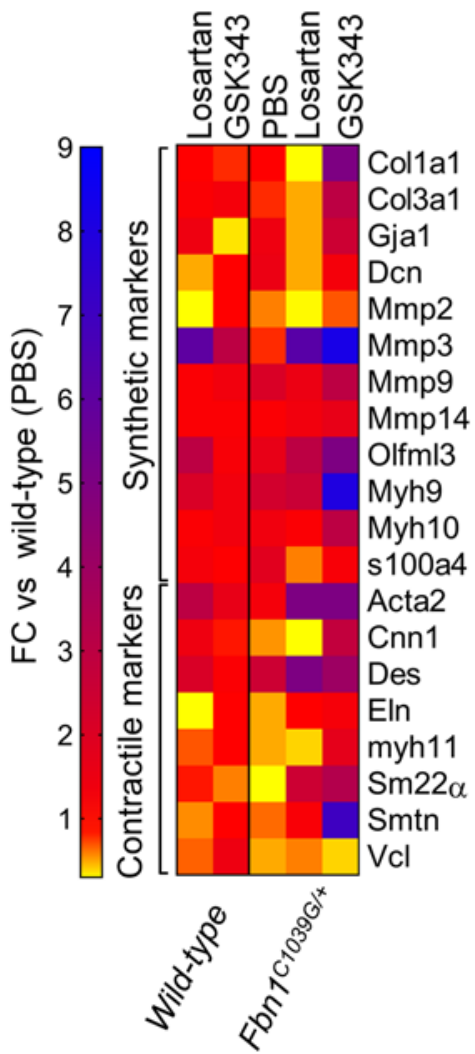

C
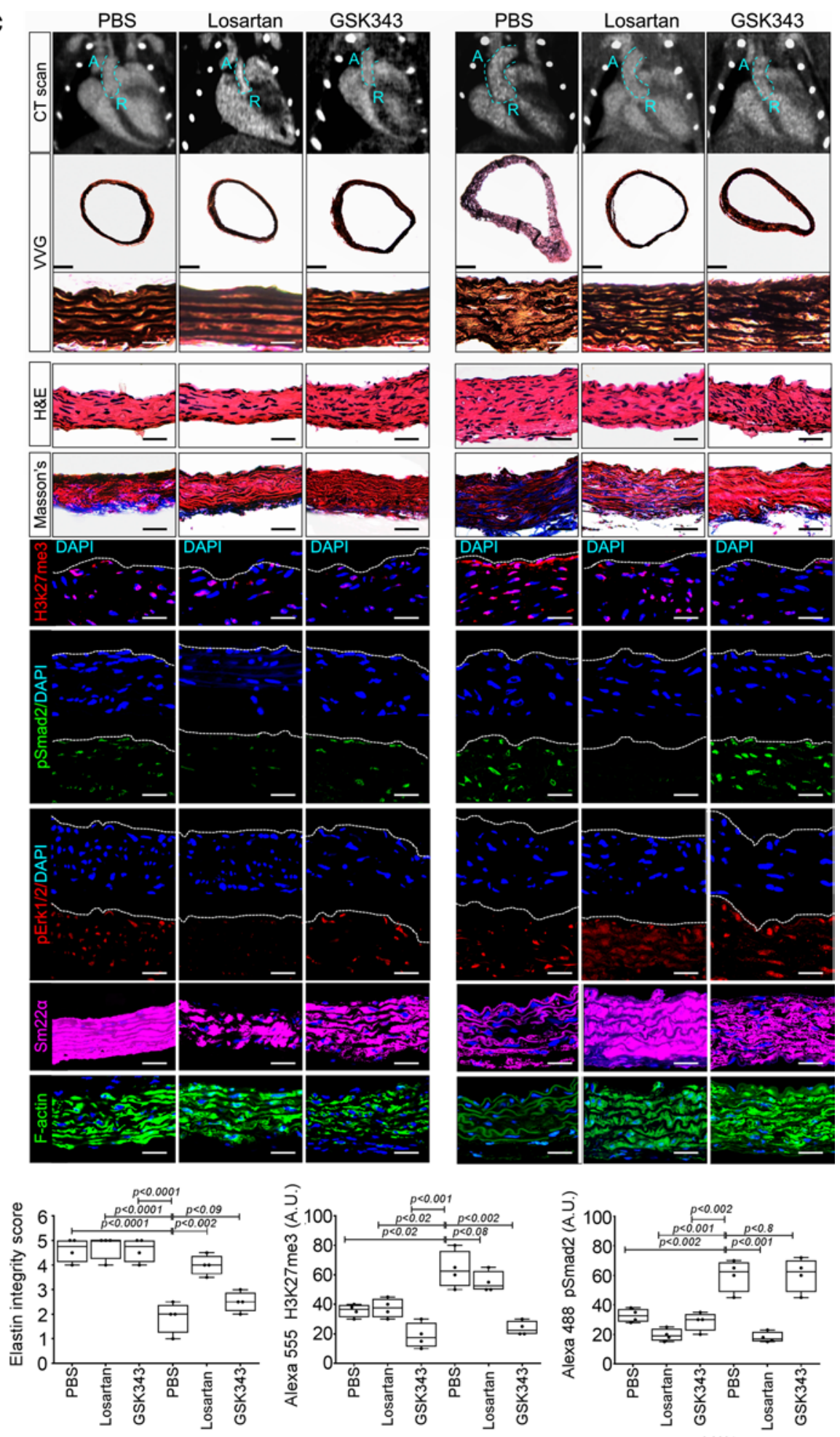

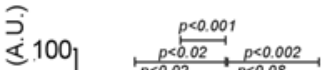

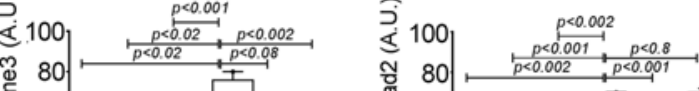

है 60.

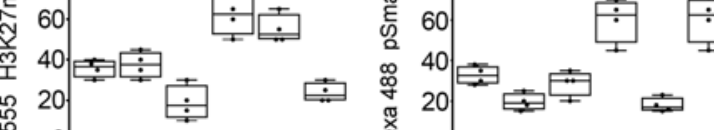

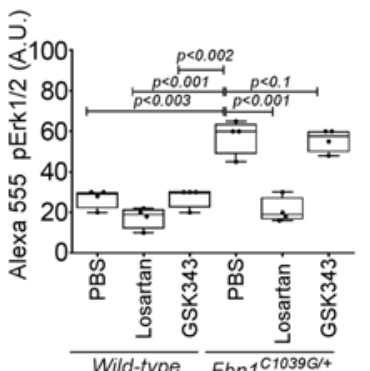

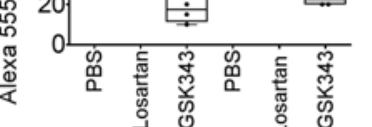

$p<0.0001$

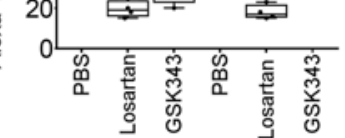

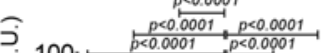

$p<0.000$

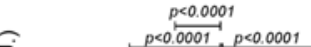

$\int_{100} \frac{p<0.0001, p<0.0001}{p<0.0001, p<0.0001}$

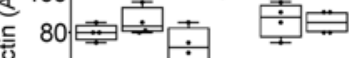

60

邑 40

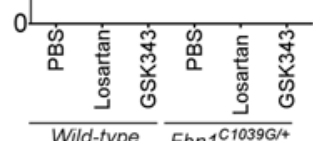

20 20 
Figure 4. In vivo inhibition of EZH2 activity palliates experimental aortic aneurysm. (A) Echocardiographic aortic root and ascending aortic quantification (at 6 months of age) of mice treated from 2 months to 6 months of age. WT (PBS, $n=8$; losartan, $n=6 ;$ GSK343, $n=6$ ) and Fbn $710396 /+(P B S$, $n=8$; losartan, $n=6$; GSK343, $n=6$ ) mice were treated (1-way ANOVA, post hoc Tukey's test) (B) Expression pattern of VSMC phenotypic markers in aortas from WT or Fbn 1 ${ }^{10396 /+}$ mice treated with losartan or GSK343 from 2-6 months of age (PBS, $n=8$; losartan, $n=6$; GSK343, $n=6$ ) and Fbn $7^{10396 /+}$ (PBS, $n=8$; losartan, $n=6$; CSK343, $n=6$ ). RNA levels were normalized to WT mice treated with PBS. (C) Top: CT scan and histologic analysis of WT or Fbn $7^{110396 /+}$ mouse aortas treated with either PBS, losartan, or GSK343. Low-magnification aorta VVG scale bar: $250 \mu \mathrm{m}$; high-magnification VVG, H\&E, Masson's trichrome, and immunofluorescence scale bars: $50 \mu \mathrm{m}$. Bottom: Quantification of histologic parameters (1-way ANOVA, post hoc Tukey's test). FC, fold change; VVG, Verhoeff-Van Gieson stain.

its inability to repress synthetic markers (Figure 4, B and C) - both losartan or GSK343 demonstrated an improvement in aortic architecture and filamentous actin content (Figure 4C). Histologic staining shows a significant suppression of H3K27me3 staining in $\mathrm{Fbn}^{\mathrm{Cl039G/+}}$ aortas from GSK343-treated animals when compared with either control or losartan-treated $\mathrm{Fbn}^{\mathrm{ClO} 103 \mathrm{G} /{ }_{+}}$mice. Interestingly, despite effective control of aortic aneurysm formation, the GSK343 compound did not suppress TGF- $\beta$ activity, as assessed by phosphorylation of Smad2 or Erk1 protein (Figure 4C). Immunostaining of aortas demonstrated recovery of SM22 $\alpha$ protein expression in both treatments when compared with control Fbn $1^{\mathrm{ClO} 039 \mathrm{G} / \mathrm{+}}$ mice (Figure $4 \mathrm{C}$ ).

We next wished to determine whether synergism of both agents could assist regression of established $\mathrm{Fbn}^{\mathrm{ClO} 103 \mathrm{G} /+}$ aortic disease. To test this hypothesis, we chose to start treatment of animals later, after established aortic enlargement. Therefore, beginning at 4 months of age, Fbn $1^{\mathrm{C} 1039 \mathrm{G} / \mathrm{+}}$ mice were treated with either losartan alone, GSK343 alone, or losartan plus GSK343 and were monitored for an additional 2 months via echocardiography. Remarkably, aortas from $F b n 1^{\mathrm{Cl039G/+}}$ mice treated with losartan plus GSK343 showed decreasing aortic dimensions (Figure 5, A and B). Histologic investigation of aortic tissue from $\mathrm{Fbn}^{\mathrm{ClO} 103 \mathrm{G} /+}$ animals demonstrates decreased collagen deposition staining in losartan-, GSK343-, or losartan plus GSK343-treated mice, while improvements in elastin integrity was noted only with losartan treatment, either alone or in combination with GSK343 (Figure 5C). Further immunostaining analysis showed restoration of Sm22 $\alpha$, Myh11, and calponin (Cnn) expression in GSK343-treated animals, either alone or in combination with losartan (Figure 5C).

\section{Discussion}

In humans, TAA is often caused by mutations in genes that affect important aspects of aortic SMC function. We undertook this study to discover how contractile protein dysregulation affects TAA. Multiple contractile proteins were found to be repressed at the transcriptional level, and in depth analysis of the locus of the TAGLN gene, encoding SM22 $\alpha$, revealed epigenetic silencing. In this study, we demonstrated that loss of SM22 $\alpha$, an abundant contractile protein, is a mediator of experimental aneurysm progression. SM22 $\alpha$ is an actin binding enzyme belonging to the $\mathrm{Cnn}$ family of proteins, known to have actin binding and actin bundling activities. It has been identified as an important modulator of actin dynamics in metastatic cancer cells (30), and in fact, multiple tumors show downregulation or deletion of the TAGLN gene $(31,32)$. Cells deficient in SM22 $\alpha$ exhibit increased motility, decreased levels of stress fiber formation, and increased expression and activity of MMPs $(29,30)$ - characteristics shared by VSMCs isolated from aneurysms. In PC3 prostate cancer cells deficient in SM22 $\alpha$, the reintroduction of expression established decreased motility and impaired ability to invade Matrigel, in part through decreased activity of MMPs (30). MMP activity has long been linked to aneurysm progression, and we and others have shown that VSMCs similarly increase this activity upon loss of SM22 $\alpha$ expression (29) (Figure 1I). These data reveal similarities between shared phenotypes in VSMCs in TAA and cancerous cells. To interpret the observed transcriptional suppression of SM22 $\alpha$ expression, we analyzed the TAGLN gene locus and found occupancy of EZH2 and associated H3K27me3 chromatin modifications (Figure 6).

EZH2 is the catalytic subunit of the polycomb repressive complex 2 (PRC2) and mediates methylation of lysine 27 of histone subunit 3 (H3K27me3), therefore silencing specific gene loci. EZH2 is recruited during development to suppress genes associated with cell fate $(33,34)$. In some scenarios, such as in cancer stem cells, EZH2 is targeted to genes associated with lineage specification and therefore acts to suppress differentiation (35). However, this generalization has exceptions, and in some cell types, EZH2 is required for the maintenance of cell lineage (36). Although we studied primarily contractile protein regulation, this mechanism may also target transcription factors required for the maintenance of VSMC identity. In our system, the observed chromatin changes correlated with an inability of transcriptional mediators, such as SMAD3, to bind to promoter sequence. Increased TGF- $\beta$ signaling, as assayed by SMAD protein phosphorylation, has been a redundant observation in human and murine TAA tissue, and suppression of TGF- $\beta$ signaling has demonstrated a ther- 
A
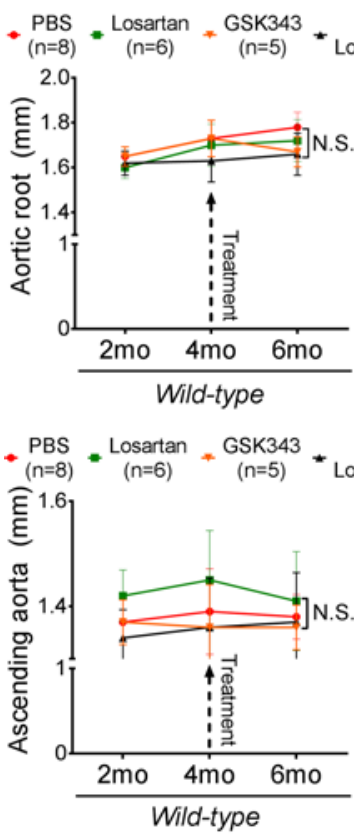

C
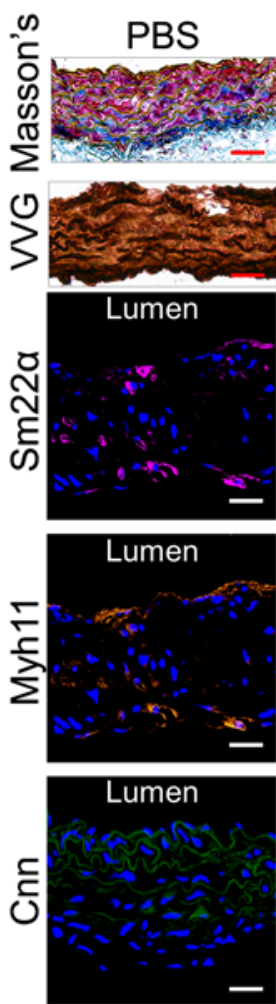

- $\underset{(n=8)}{\text { PBS }}=\underset{(n=6)}{\text { Losartan }} * \underset{(n=5)}{\operatorname{GSK} 343} * \underset{\text { Losartan }(n=5)}{\text { GSK343/ }}$

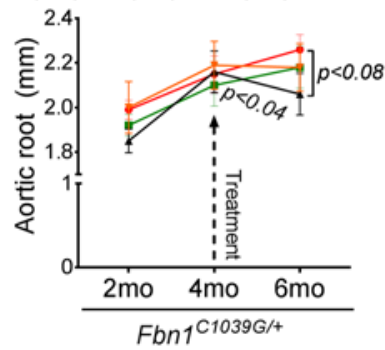

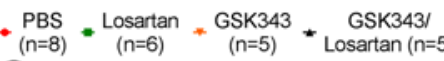

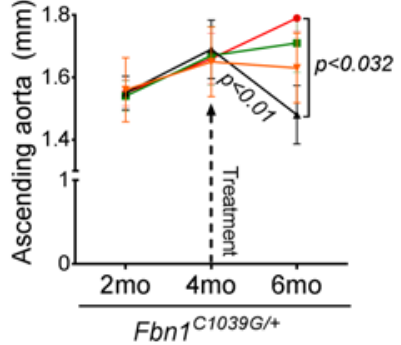

B

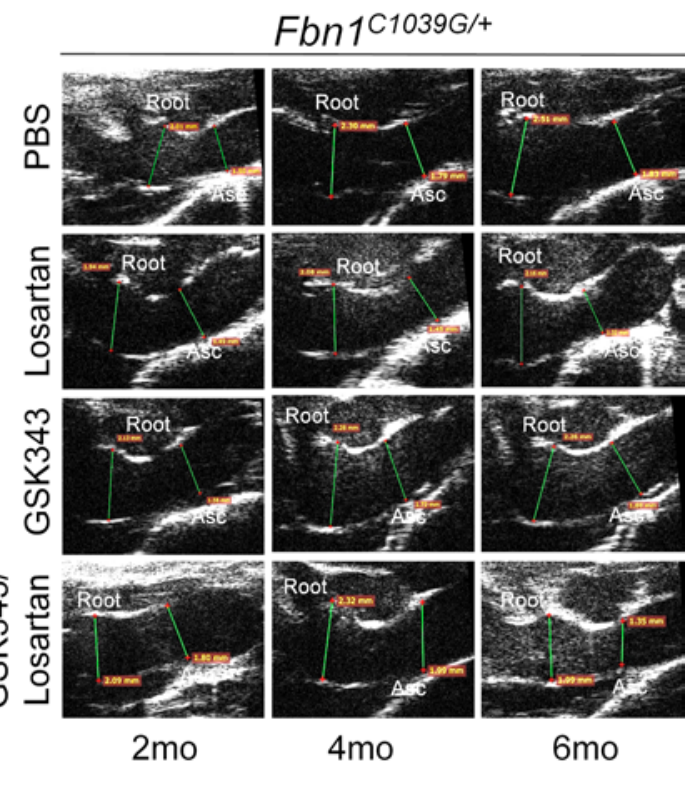



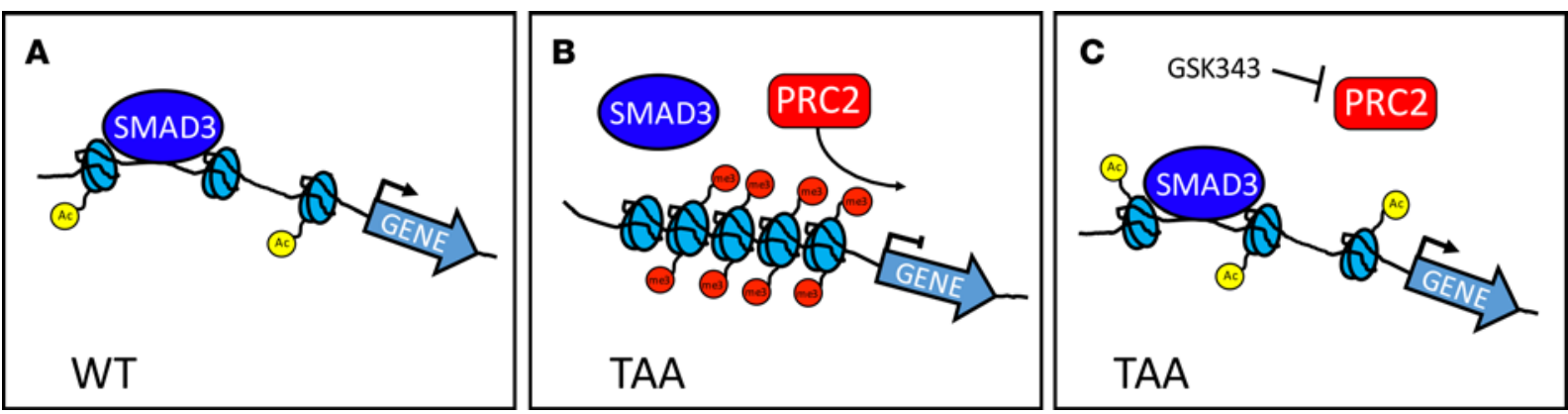

Figure 6. Schematic representation of epigenetic repression in TAA. (A) Contractile genes (e.g., TAGLN, MYH11, SMTN) responsible for aortic homeostasis are actively transcribed in VSMCs through the action of transcription factors such as SMAD3, among others. (B) In thoracic aortic aneurysms, the EZH2-containing polycomb repressive complex 2 (PRC2) mediates addition of histone 3 lysine 27 trimethylation marks to the promoter and gene body of aortic genes, preventing transcription factor access to chromatin. (C) Treatment with the EZH2 inhibitor GSK343 derepresses contractile protein expression by allowing transcription factors (such as SMAD3) access to chromatin.

apeutic response in experimental aneurysm in the $F b n 1^{\mathrm{ClO3gG/+}}$ mouse model $(24,37)$. However, similar suppression of TGF- $\beta$ signaling with anti-TGF- $\beta$ neutralizing antibodies exacerbated aortic disease in angiotensin 2 -infused mice (38). Furthermore, loss-of-function mutations in canonical TGF- $\beta$ mediators such as TGFB2 and $S M A D 3$ underlie syndromic aortic disease, but aortic tissue from patients with these mutations consistently shows upregulation of TGF- $\beta$ signaling. These seeming contradictions of the role of TGF- $\beta$ in aortic aneurysm etiology and progression continue to be controversial (39). Differential transcriptional responsiveness caused by epigenetic silencing such as described in this study may help to explain some, but not all, of these paradoxical observations. These data offer a paradigm where epigenetic modifications in TAA prevent normal transcriptional responsiveness in critical genes that would otherwise improve aortic homeostasis in the context of increased TGF- $\beta$ activity, routinely observed in TAA samples. Deficient canonical TGF- $\beta$ signaling may induce epigenetic remodeling of VMSCs and set the stage for compensatory upregulation of TGF- $\beta$ signaling during aneurysm progression. Some effects downstream of TGF- $\beta$ signaling, including fibrotic cascades (mediating arterial stiffness) and MMP upregulation (mediating extracellular matrix destruction), are harmful to aortic homeostasis, and antagonism of such products may improve performance - hence, a rationale for TGF- $\beta$ antagonism. However, VSMCs are sensitive to intrinsic inhibition of TGF- $\beta$ signaling, as demonstrated in models that genetically inhibit members of the canonical signaling cascade (such as experiments in Tgfbr2-deficient mice) (40-42) or human conditions mediated by loss-of-function mutations in TGF- $\beta$ mediators $(13,14,16,17)$. Therefore, restoration of intrinsic TGF- $\beta$ signaling activity within VSMCs in TAA may also prove beneficial. Simple models of TGF- $\beta$ signaling as either simply harmful or beneficial fail to incorporate the complexity of compensatory signaling events in VSMC biology. Epigenetic silencing of contractile loci may explain the ineffectual nature of TGF- $\beta$ signaling in improving contractile protein expression (SM22 $\alpha, \alpha$-SMA, and smMHC, among others) in aortic aneurysms. This type of mechanism is more relevant to the ascending aortic aneurysm, which has a unique clinical profile even in the absence of Mendelian association (43) - a mechanistic correlate that has been noted in the differential susceptibility to intrinsic VSMC TGF- $\beta$ inhibition in model systems - when compared with descending aortic VSMCs (42).

The observation that EZH2 inhibition allows for more efficient expression of SM22 $\alpha$, a protein necessary for proper aortic homeostasis (Figure 2), suggested the opportunity to use this agent as a therapeutic agent to treat aortic aneurysm. To this end, we treated experimental aortic aneurysm in $F b n 1^{\mathrm{ClO} 103 \mathrm{G} / \mathrm{H}}$ mice and found a therapeutic response comparable with therapy with losartan. The effect seems to work through a distinct molecular mechanism. TGF- $\beta$ signaling, as assessed by suppression of smad 2 phosphorylation, was reduced in losartan-treated aortic tissue but not in GSK343-treated animals. Conversely, GSK343 significantly decreased aortic medial H3K27me3 modifications, while losartan did not. These observations led us to investigate combinatorial therapy, which offered additional benefits (Figure 5). The clinical benefits of using combinatorial therapies for chronic disease are clear for other cardiovascular diseases such as heart failure, hyperlipidemia, and diabetes, and such may be the case in TAA. Despite this promise, EZH2 inhibitors would be expected to have major off-target biologic effects, especially if their use is contemplated for a chronic condition such as aneurysm. For this reason, therapeutic strategies hoping to take advantage of pathogenic epigenetic events will require agents with greater target specificity or more precise tissue-targeting properties. 
In general, medical therapy for TAA is limited to different classes of antihypertensive medications, some with anti-TGF- $\beta$ activity such as angiotensin II receptor blockers (ARBs) or angiotensin-converting enzyme inhibitors (ACE inhibitors). This proof-of-concept experiment, demonstrating a therapeutic effect of a chromatin-modifying agent, suggests that unsuspected classes of medication may have a role in the future medical treatment of TAA.

\section{Methods}

Aortic samples. Aortic samples were collected from patients undergoing cardiac surgery at the MGH. Upon tissue collection, the media layer was dissected and stored at $-80^{\circ} \mathrm{C}$ for analysis of RNA and protein expression. Control aortic tissue was obtained from patients undergoing orthotopic cardiac transplant. IRB permissions do not allow for demographic information from discarded tissue to be collected or stored.

Primary aortic SMC lines. Primary human aortic SMCs (HAoSMC) from healthy donors were purchased from Cell Applications Inc. (catalog 354K-05a). Primary aneurysm aortic SMCs were isolated from fresh TAA tissue at the moment of surgery by standard explant of the aortic media. Mouse aortic SMCs were isolated by standard explant of the ascending section of the aortas from WT or Fbn $1^{\mathrm{C} 1039 \mathrm{G} / \mathrm{+}}$ mice. SMC identity was assessed by immunofluorescence staining of contractile markers, including SM $22 \alpha$, Cnn, smoothelin, and vinculin. In order to preserve cell identity, all experiments were carried out at passages 1-5. Human and murine SMCs were grown with SMC growth medium from Cell Applications Inc. (catalog 311-500). See complete unedited blots in the supplemental material.

$R N A$ extraction and $q P C R$ analysis. Total RNA was prepared from tissue or primary cells using RNeasy kit (Qiagen) following the manufacturer's protocol. The cDNA was prepared by reverse transcription, and expression of SMC contractile genes were analyzed by qPCR on SYBR green system (Applied Biosystem). Plasma from WT or Fbn $1^{C 1039 G /+}$ mice $(50 \mu \mathrm{l})$ were used to prepare total RNA, as mentioned above. Expression results were analyzed by the $\triangle \triangle \mathrm{CT}$ method, and GAPDH (encoding glyceraldehyde-3-phosphate dehydrogenase) was used as a housekeeping gene. FCs were calculated by taking the average over all of the control samples as the baseline. Plasmatic levels of the murine Tagln (SM22 $\alpha$ ) transcript were measured using $100 \mu$ of plasma in $2 \mathrm{ml}$ of sterile PBS (Gibco), followed by 2 hours of ultracentrifugation at $100,000 \mathrm{~g}$. The supernatant was removed, and the vesicular fraction was resuspended with $750 \mu \mathrm{l}$ of TRIzol reagent followed by RNA extraction using the RNeasy kit (Qiagen) according to the manufacturer's protocol. We settled on GAPDH as the most stable housekeeping gene, both in healthy SMCs and in our plasma samples, through direct testing under our experimental conditions. To achieve this observation, we ran qPCR assays using the RT2Profiler PCR Array from Qiagen (human catalog 330231/PAHS-055Z and mouse catalog 330231/PAMM-055Z). This array contains 5 commonly used housekeeping genes such ACTB ( $\beta$-actin), B2M ( $\beta$-2 microglobulin, GAPDH (Glyceraldehyde-3-phospho dehydrogenase), HPRT1 hypoxanthine phosphoribosyltransferase 1, RPLP0 (Ribosomal protein, large, P0). Additionally, the arrays contain 1 human genomic DNA contamination control, 3 reverse transcription controls, and 3 positive PCR controls. PCR primers and siRNAs are listed in Supplemental Table 2.

ChIP-qPCR. For ChIP-qPCR from human aortic tissue, $200 \mathrm{mg}$ of frozen sectioned aortic media from controls or TAA donors were fixed with $1 \%$ of formaldehyde at $37^{\circ} \mathrm{C}$ for 20 minutes, quenched with $125 \mathrm{mM}$ glycine for 5 minutes at room temperature (RT), and supplemented with $1 \times$ of protease inhibitor cocktail (Roche Diagnostics), followed by preparation of total protein lysates. Next, $50 \mu \mathrm{g}$ of total protein lysates were sonicated to shear chromatin to an average length of $500-1,500 \mathrm{bp}$, followed by centrifugation for 10 minutes at max speed. Supernatants were collected in a 2-ml tube containing $6 \mu \mathrm{g}$ of monoclonal antibodies against EZH2 (clone AC22, Active Motif) or H3K27me3 (Abcam, ab4729) and $1 \mathrm{ml}$ of lysis buffer supplemented with $1 \times$ of protease inhibitor cocktail (Roche Diagnostics), followed by incubation overnight (14 hours) at $4^{\circ} \mathrm{C}$. Immunoprecipitates were analyzed using EpiTect ChIP kit according to the manufacturer's instructions (Qiagen). Primers used to scan the promotor and gene body of TAGLN gene are listed in Supplemental Table 1. The TAGLN gene intronic regions 1.3 and 1.6 were analyzed for consensus sequence binding motifs using Genomatix software. For ChIP-qPCR from cells, 5 million cells from healthy or isolated aortic aneurysm VSMCs were first treated with $0.1 \%$ of DMSO or $10 \mathrm{ng} / \mathrm{ml}$ of human recombinant TGF- $\beta 1$ (Abcam) for 24 hours in growing medium. Then, cells were fixed with $1 \%$ of formaldehyde at $37^{\circ} \mathrm{C}$ for 10 minutes and quenched with $125 \mathrm{mM}$ glycine for 5 minutes at RT, followed by preparation of total protein lysates. Next, monoclonal antibodies against SMAD3 were incubated with $20 \mu \mathrm{g}$ of lysates and analyzed as described above. PCR primers, siRNAs, and antibodies are listed in Supplemental Table 2. 
siRNA inhibition. Healthy VSMCs were transfected with $30 \mathrm{nM}$ of siCtrl, siSMAD3, or siSM22 $\alpha$ for 48 hours with lipofectamine 2000 (Thermo Fisher Scientific) at $5 \mu 1 / \mathrm{ml}$, followed by 24 hours of normal growing medium. Then, $30 \mu \mathrm{g}$ of total protein was prepared from siRNA-treated cells to analyze the silencing of targeted transcripts by immunoblotting or nucleic acid analysis. siRNAs are listed in Supplemental Table 2.

MMP activity. For in vitro MMP activity, healthy VSMCs were transfected with $30 \mathrm{nM}$ of siCtrl or siSM22 $\alpha$, as described above. Proteins (30 $\mu \mathrm{g}$ of total) were prepared from untransfected, siCtrl, and siSM22 $\alpha$-treated cells to measure the MMP activity by gelatin zymography and were analyzed by ImageJ software (NIH). For in vivo MMP activity assay, mice were tail-vein injected with $300 \mu 1$ of MMPSense 680 FAST, a near-infrared fluorescence sensor for MMP2 and MMP9 activity from PerkinElmer. Mice were sacrificed 24 hours after injection, and aortas were dissected and analyzed using a Kodak image station 4000MM Pro for macroscopic fluorescence reflectance molecular imaging (44).

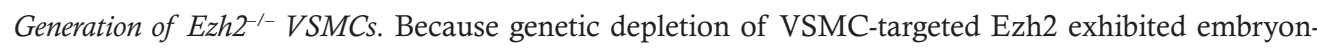
ic lethality, we crossed $F b n 1^{C 1093 G /+}$ to $E z h 2^{f l f l}$ mice (The Jackson Laboratory, Ezh2tm2Sho/J; catalog 022616). Then, ascending aortas of $F b n 1^{11093 G /+} E z h 2^{f / f l}$ or $E z h 2^{f / f l}$ mice at 4 month of age were isolated as described above. At passage 1, isolated VSMC cells were transduced with lentivirus overexpressing CMV-cre to deplete Ezh2 gene expression (pFUGW-H1 empty vector was a gift from Sally Temple; Addgene plasmid 25870). Following 72 hours of infection, cells were incubated with normal growing media for 24 hours before TGF- $\beta 1$ stimulation as described above.

Overexpression of a high-chromatin affinity EZH2 isoform. Replacement of the serine 21 by Alanine in the EZH2 (EZH2S21A) protein induces 2- to 4-fold more affinity to bind with histone 3 than its WT counterpart (45). The pcDNA3-3myc-6His-EZH2 21A construct was acquired from Addgene (plasmid 42663, Laboratory of Mien-Chie Hung, MD Anderson Cancer Center, Houston, Texas). Plasmid (1-2 $\mu \mathrm{g})$ was transiently transfected into human SMCs using lipofectamine TLX (Thermo Fisher Scientific) and opti-MEM medium (Thermo Fisher Scientific) for 48 hours. Then, cells were supplied with fresh growing media containing recombinant TGF- $\beta 1(10 \mathrm{ng} / \mathrm{ml})$ overnight (14 hours).

Echocardiograms and micro-CT scan. Dimensions from each animal represent averages of measurements made on still frames in systole of the maximal internal diameter of the aortic valve annulus, aortic sinuses, sinotubular junction, or ascending aorta by a cardiologist blinded to genotype. The aorta was imaged using a standard parasternal long-axis view. Based on historic controls, the mean diameter of the WT aortic root at 6 months of life is $1.76 \pm 0.2 \mathrm{~mm}$, while in $\mathrm{Fbn} 1^{\mathrm{C} 1039 \mathrm{G} / \mathrm{+}}$ mice, the mean diameter is $2.22 \pm 0.25 \mathrm{~mm}$. This gives a window to observe therapeutic improvement of $0.46 \mathrm{~mm}$. Nair hair removal cream was used on all mice the day prior to echocardiograms. All echocardiograms were performed on awake, unsedated mice using the Visualsonics Vevo imaging system and a $30 \mathrm{MHz}$ transducer. All CT images were acquired using an Inveon small animal micro CT (Siemens Medical Solutions Inc.). All mice received i.v. iodinated contrast (Isovue-370, Bracco Diagnostic Inc.) at $20 \mu \mathrm{l} / \mathrm{min}$ during the scan. CT images had an isometric voxel of $0.11 \mathrm{~mm}$. Images were visualized and analyzed in OsiriX software.

Histology. Latex was injected into the left ventricular apex under low pressure until it was visible in the femoral artery. Animals were then fixed in formalin (10\%) for 24 hours before transfer to $70 \%$ ethanol for dissection and storage. Aortas were then removed from the animals or dissected in situ for photography prior to paraffinization and sectioning $(10 \mu \mathrm{M})$. Slides were produced for tissue staining or stained with standard stains including H\&E, collagen deposition (Trichrome Stain Kit, MilliporeSigma), elastin (Verhoeff-Van Gieson, Thermo Fisher Scientific), or F-actin (ActinGreen 488 ReadyProbes, Thermo Fisher Scientific) for quantitative analysis. Elastin integrity score was rated by blinded observers and graded on an arbitrary scale of 5 (indicating high-quality elastic fiber) to 1 (indicating severe elastin fragmentation). For nuclear staining, aortas from human and mice were cryosectioned using OCT standard protocol.

In vitro GSK343 treatment. VSMCs at 60\%-70\% confluence were supplemented with opti-MEM 24 hours before treatment with complete growing media containing GSK343 at a concentration of $10 \mu \mathrm{M}$ for 4 days. Fresh media containing GSK343 was supplied daily. For GSK343/TGF- $\beta 1$ (10 ng/ml) combo treatment, cells were first treated with complete media containing GSK343 at a concentration of $10 \mu \mathrm{M}$ during the 2 first days, followed by GSK343/TGF- $\beta 1$ combination treatment for the next 2 days. Prior to cellular collection, cells were washed with sterile PBS, and RNA or protein extraction were performed as described above.

In vivo drug treatments. Cohorts of 8 -week-old WT or Fbn $1^{\mathrm{C1039G/+}}$ mice were treated with oral administration in drinking water of either losartan or GSK343 for 16 weeks (Figure 4). Losartan was dissolved in drinking water and filtered to reach a concentration of $0.6 \mathrm{~g} / 1$, giving an estimate of $60 \mathrm{mg} /$ 
$\mathrm{Kg}$ /day, as described previously. GSK343 inhibitor (25 mg)was dissolved in $1 \mathrm{~mL}$ of DMSO to obtain a stock solution of $46 \mathrm{mM}$. Then, $200 \mathrm{ml}$ of drinking water was supplemented with $250 \mu 1$ of GSK343 stock solution. Control group for either drug treatment was given with equal volumes of sterile water. Bottles containing either losartan or GSK343 were replaced with fresh water supplied with drugs every 3 days. For experiments described in Figure 5, 16-week-old WT or Fbn $1^{\text {C1039G/+ }}$ mice were treated for 8 weeks, as described above. Additionally, a group of mice were given a combination of both losartan and GSK343 at the same concentrations as of other single-drug treatments. Aortic dimensions were monitored monthly by echocardiograms as mentioned above.

Statistics. Results are given as mean \pm SD Student's $t$ test (2-tailed) was applied to determine the statistical significance of difference between control and treated groups $\left({ }^{*} P<0.05,{ }^{* *} P<0.01\right.$ and $\left.{ }^{* *} P<0.001\right)$. For all experiments, at least 3 experimental replicates were performed. Least squares method was used to fit line in Figure 1F. Scatter graphs show mean \pm SD. Data were analyzed and graphs were prepared with Prism 6.0 (GraphPad Software). Differences between multiple groups (echocardiography and histologic analysis) were assessed and $P$ values generated by 1 -way ANOVA followed by Tukey's honestly significance difference (HSD) post hoc test ( $95 \% \mathrm{CI}$ is plotted). $P$ values of less than 0.05 were considered statistically significant.

Study approval. All experimental procedures were approved by the IACUC of the MGH. Human samples were obtained under a protocol approved by the Partners HealthCare IRB, funded by MGH.

\section{Author contributions}

MEL and CLLC designed studies. MEL, CLLC, CWK, FAJ, and CM performed experiments. MEL, ASJ, EMI, and CLLC acquired samples and analyzed data. MEL and CLLC wrote the manuscript.

\section{Acknowledgments}

MEL is supported by the Fredman Fellowship, the Toomey Fund for Aortic Dissection Research, and the Hassenfeld Fellowship. MEL, CLLC, and CM are supported by HL130113.

Address correspondence to: Mark E. Lindsay, Harvard Medical School, Richard B. Simches Research Building, Room 3212, 185 Cambridge Street, Boston, Massachusetts 02114, USA. Phone: 617.643.3458; Email: Lindsay.Mark@mgh.harvard.edu.

1. Grewal N, et al. Ascending aorta dilation in association with bicuspid aortic valve: a maturation defect of the aortic wall. $J$ Thorac Cardiovasc Surg. 2014;148(4):1583-1590.

2. Ailawadi G, et al. Smooth muscle phenotypic modulation is an early event in aortic aneurysms. J Thorac Cardiovasc Surg. 2009;138(6):1392-1399.

3. Inamoto S, et al. TGFBR2 mutations alter smooth muscle cell phenotype and predispose to thoracic aortic aneurysms and dissections. Cardiovasc Res. 2010;88(3):520-529.

4. Shen J, et al. Disruption of SM22 promotes inflammation after artery injury via nuclear factor kappaB activation. Circ Res. 2010;106(8):1351-1362.

5. Feil S, Hofmann F, Feil R. SM22alpha modulates vascular smooth muscle cell phenotype during atherogenesis. Circ Res. 2004;94(7):863-865.

6. Dong LH, et al. TRAF6-Mediated SM22 $\alpha$ K21 Ubiquitination Promotes G6PD Activation and NADPH Production, Contributing to GSH Homeostasis and VSMC Survival In Vitro and In Vivo. Circ Res. 2015;117(8):684-694.

7. Rinaldi B, et al. G-CSF contributes at the healing of tunica media of arteriotomy-injured rat carotids by promoting differentiation of vascular smooth muscle cells. J Cell Physiol. 2016;231(1):215-223.

8. Hibino N, et al. Potential Molecular Mechanism of Retrograde Aortic Arch Stenosis in the Hybrid Approach to Hypoplastic Left Heart Syndrome. Ann Thorac Surg. 2015;100(3):1013-1020.

9. Guo DC, et al. Mutations in smooth muscle alpha-actin (ACTA2) lead to thoracic aortic aneurysms and dissections. Nat Genet. 2007;39(12):1488-1493.

10. Zhu L, et al. Mutations in myosin heavy chain 11 cause a syndrome associating thoracic aortic aneurysm/aortic dissection and patent ductus arteriosus. Nat Genet. 2006;38(3):343-349.

11. Guo DC, et al. Recurrent gain-of-function mutation in PRKG1 causes thoracic aortic aneurysms and acute aortic dissections. Am J Hum Genet. 2013;93(2):398-404.

12. Wang L, et al. Mutations in myosin light chain kinase cause familial aortic dissections. Am J Hum Genet. 2010;87(5):701-707.

13. Loeys BL, et al. A syndrome of altered cardiovascular, craniofacial, neurocognitive and skeletal development caused by mutations in TGFBR1 or TGFBR2. Nat Genet. 2005;37(3):275-281.

14. Lindsay ME, et al. Loss-of-function mutations in TGFB2 cause a syndromic presentation of thoracic aortic aneurysm. Nat Genet. 2012;44(8):922-927.

15. Bertoli-Avella AM, et al. Mutations in a TGF- $\beta$ ligand, TGFB3, cause syndromic aortic aneurysms and dissections. $J$ Am Coll Cardiol. 2015;65(13):1324-1336. 
16. van de Laar IM, et al. Mutations in SMAD3 cause a syndromic form of aortic aneurysms and dissections with early-onset osteoarthritis. Nat Genet. 2011;43(2):121-126

17. Boileau C, et al. TGFB2 mutations cause familial thoracic aortic aneurysms and dissections associated with mild systemic features of Marfan syndrome. Nat Genet. 2012;44(8):916-921.

18. Isselbacher EM, Lino Cardenas CL, Lindsay ME. Hereditary Influence in Thoracic Aortic Aneurysm and Dissection. Circulation. 2016;133(24):2516-2528.

19. Qiu P, Feng XH, Li L. Interaction of Smad3 and SRF-associated complex mediates TGF-beta1 signals to regulate SM22 transcription during myofibroblast differentiation. J Mol Cell Cardiol. 2003;35(12):1407-1420.

20. Zhang P, et al. Smad4 Deficiency in Smooth Muscle Cells Initiates the Formation of Aortic Aneurysm. Circ Res. 2016;118(3):388-399.

21. Chen S, Kulik M, Lechleider RJ. Smad proteins regulate transcriptional induction of the SM22alpha gene by TGF-beta. Nucleic Acids Res. 2003;31(4):1302-1310.

22. Qiu P, Ritchie RP, Gong XQ, Hamamori Y, Li L. Dynamic changes in chromatin acetylation and the expression of histone acetyltransferases and histone deacetylases regulate the SM22alpha transcription in response to Smad3-mediated TGFbeta1 signaling. Biochem Biophys Res Commun. 2006;348(2):351-358.

23. Renard M, et al. Altered TGFbeta signaling and cardiovascular manifestations in patients with autosomal recessive cutis laxa type I caused by fibulin-4 deficiency. Eur J Hum Genet. 2010;18(8):895-901.

24. Habashi JP, et al. Losartan, an AT1 antagonist, prevents aortic aneurysm in a mouse model of Marfan syndrome. Science. 2006;312(5770):117-121.

25. Rensen SS, Doevendans PA, van Eys GJ. Regulation and characteristics of vascular smooth muscle cell phenotypic diversity. Neth Heart J. 2007;15(3):100-108.

26. Zhang L, Yu C, Chang Q, Luo X, Qiu J, Liu S. Comparison of gene expression profiles in aortic dissection and normal human aortic tissues. Biomed Rep. 2016;5(4):421-427.

27. Sun Y, et al. The regulatory role of smooth muscle 22 on the proliferation of aortic smooth muscle cells participates in the development of aortic dissection. J Vasc Surg. 2017;66(3):875-882.

28. Lesauskaite V, et al. Smooth muscle cells of the media in the dilatative pathology of ascending thoracic aorta: morphology, immunoreactivity for osteopontin, matrix metalloproteinases, and their inhibitors. Hum Pathol. 2001;32(9):1003-1011.

29. Nair RR, Solway J, Boyd DD. Expression cloning identifies transgelin (SM22) as a novel repressor of 92-kDa type IV collagenase (MMP-9) expression. J Biol Chem. 2006;281(36):26424-26436.

30. Thompson O, Moghraby JS, Ayscough KR, Winder SJ. Depletion of the actin bundling protein SM22/transgelin increases actin dynamics and enhances the tumourigenic phenotypes of cells. BMC Cell Biol. 2012;13:1.

31. Yamamura H, Yoshikawa H, Tatsuta M, Akedo H, Takahashi K. Expression of the smooth muscle calponin gene in human osteosarcoma and its possible association with prognosis. Int J Cancer. 1998;79(3):245-250

32. Prasad PD, Stanton JA, Assinder SJ. Expression of the actin-associated protein transgelin (SM22) is decreased in prostate cancer. Cell Tissue Res. 2010;339(2):337-347.

33. Boyer LA, et al. Polycomb complexes repress developmental regulators in murine embryonic stem cells. Nature. 2006;441(7091):349-353.

34. Ezhkova E, et al. Ezh2 orchestrates gene expression for the stepwise differentiation of tissue-specific stem cells. Cell. 2009;136(6):1122-1135

35. Chang CJ, et al. EZH2 promotes expansion of breast tumor initiating cells through activation of RAF1- $\beta$-catenin signaling. Cancer Cell. 2011;19(1):86-100.

36. Wang L, Jin Q, Lee JE, Su IH, Ge K. Histone H3K27 methyltransferase Ezh2 represses Wnt genes to facilitate adipogenesis. Proc Natl Acad Sci USA. 2010;107(16):7317-7322.

37. Renard M, et al. Novel MYH11 and ACTA2 mutations reveal a role for enhanced TGF $\beta$ signaling in FTAAD. Int J Cardiol. 2013;165(2):314-321.

38. Wang Y, et al. TGF-beta activity protects against inflammatory aortic aneurysm progression and complications in angiotensin II-infused mice. J Clin Invest. 2010;120(2):422-432.

39. Mallat Z, Ait-Oufella H, Tedgui A. The Pathogenic Transforming Growth Factor- $\beta$ Overdrive Hypothesis in Aortic Aneurysms and Dissections: A Mirage? Circ Res. 2017;120(11):1718-1720.

40. Li W, et al. Tgfbr2 disruption in postnatal smooth muscle impairs aortic wall homeostasis. J Clin Invest. 2014;124(2):755-767.

41. Gallo EM, et al. Angiotensin II-dependent TGF- $\beta$ signaling contributes to Loeys-Dietz syndrome vascular pathogenesis. $J$ Clin Invest. 2014;124(1):448-460

42. Angelov SN, Hu JH, Wei H, Airhart N, Shi M, Dichek DA. TGF- $\beta$ (Transforming Growth Factor- $\beta$ ) Signaling Protects the Thoracic and Abdominal Aorta From Angiotensin II-Induced Pathology by Distinct Mechanisms. Arterioscler Thromb Vasc Biol. 2017;37(11):2102-2113

43. Vapnik JS, et al. Characteristics and Outcomes of Ascending Versus Descending Thoracic Aortic Aneurysms. Am J Cardiol. 2016;117(10):1683-1690.

44. Chang K, et al. Pioglitazone suppresses inflammation in vivo in murine carotid atherosclerosis: novel detection by dual-target fluorescence molecular imaging. Arterioscler Thromb Vasc Biol. 2010;30(10):1933-1939.

45. Cha TL, et al. Akt-mediated phosphorylation of EZH2 suppresses methylation of lysine 27 in histone H3. Science. 2005;310(5746):306-310. 